Clinical guidelines for treating caries in adults following a minimal intervention policy

- Evidence and consensus based report -

Y Momoi, M Hayashi, M Fujitani, M Fukushima, S Imazato, S Kubo, T Nikaido, A Shimizu, M Unemori, C Yamaki

Short title: Guidelines for treating caries with MI policy

The Japanese Society of Conservative Dentistry

1-43-9, Komagome, Toshima-ku, Tokyo 170-0003, Japan

Correspondence to: Mikako Hayashi, DDS, Ph.D.

Department of Restorative Dentistry and Endodontology,

Osaka University Graduate School of Dentistry,

1-8 Yamadaoka, Suita, Osaka 565-0871, Japan.

Tel: +81-6-6879-2928

Fax: +81-6-6879-2928

E-mail: mikarin@dent.osaka-u.ac.jp

Keywords: caries, minimal intervention, guideline, evidence, consensus, clinical questions 


\title{
Clinical guidelines for treating caries in adults following a minimal intervention policy \\ - Evidence and consensus based report -
}

\begin{abstract}
Objectives: In 2002, FDI (World Dental Federation) published a policy advocating that caries be treated by minimal intervention (MI). This MI policy has been accepted worldwide and is taught in universities. But acceptance in general dental practice has been slower, especially in Japan where healthcare payment and practice favor drilling and filling. To help disseminate this MI policy into general practice, the Japanese Society of Conservative Dentistry developed an evidence-based clinical Guideline for restoring carious permanent teeth in adult patients.
\end{abstract}

Methods: The Guideline was developed by a committee of nine university clinicians and a librarian. The committee selected the most frequent clinical questions in treating caries and used electronic databases to search and assess the best scientific evidence for each. Members then added their clinical experience and used the Delphi Method to discuss and reach consensus on each question on treating caries with MI policy. Graded recommendations and guidance were made for each clinical question. The provisional Guideline was strengthened after review and discussion with university researchers and general practitioners.

Results: The Guideline addresses the 16 most frequent clinical questions in treating adult caries, including restorative methods and how to tackle root caries. Recommendations for treatment using MI policy were developed using the best scientific evidence and consensus of experienced clinicians.

Clinical Significance: The Guideline offers a practical expert view of treating caries with the MI policy that incorporates the best scientific evidence, the latest techniques, the most preferable materials and the general consensus of expert clinicians. 


\section{Clinical guidelines for treating caries following a minimal intervention policy - Evidence and consensus based report -}

\section{Introduction}

Purpose and objectives

The Japan Society for Conservative Dentistry offers this Guideline as a practical aid for dentists to apply the latest diagnostic and treatment efforts in their vital quest to maintain natural teeth and enhance occlusal and masticatory functions, thus improving patients' quality of life. Japan is already on the frontline of a hyper-aging society with all the concomitant challenges for society, healthcare and budget. The government, supported by the Japan Dental Association, has set a target of " $80-20$ " - meaning that at the age of 80 a person should still have at least 20 natural teeth - and this Guideline uses evidence and consensus, along with the principles of Minimal Intervention (MI) ${ }^{1}$, and suggests clinical practice that will promote this aim.

\section{Background to the development of the Guideline}

Dentistry has made remarkable advances recently, especially in cariology, and in improved dental materials and techniques. New methods of treatment of caries actively incorporating these advances have been developed, with a strong body of evidence in support of their success. Achievement of the important 80-20 goal would be greatly helped by increased use of these new treatments based on the concept of MI and diminished use of traditional drilling and filling treatment, which often involve removal of large amounts of healthy tooth structure. Nevertheless, MI treatment of caries has often been done without relying on the substantial body of evidence of best methods and practices. The result is that different, sometimes conflicting, treatments have been chosen, leading to considerable confusion among students and clinical interns in dental educational institutions, not to speak of confusion and fear on the part of patients receiving treatment. In the traditional treatment of caries in Japan, there has been acute awareness that the government health insurance scheme rewards repeated drilling and filling. The government should encourage remuneration of diagnosis and preventative treatments.

\section{Principal premises of the Guideline}

The guidelines: 
- to offer the best patient-centered treatments of caries.

- to use a policy of minimal intervention MI.

- to set out the levels of clinical evidence wherever possible, and follow the grading system recommended by Minds ${ }^{2}$.

- to show the recommendations and guidance listed by grade that offer the best long-term prognoses in treatment of caries; the recommendations were made by the working group in the light of best available scientific evidence and their own experience, and more than 100 hours of consensus discussions and consultations with general practitioners and experts.

- to seek maximum preservation of dental pulp subjacent to extremely deep caries.

- to suggest how to remineralize root surface caries, as frequently found in elderly patients and denture wearers.

Caries is common in all age groups, but recently there has been increasing risk among elderly people. Modern dentistry has greatly advanced in the use of new restorative materials and techniques. One of the main intentions of The Japan Society for Conservative Dentistry in forming the committee was to provide these benefits to as many people as possible to achieve the 80-20 objective: this Guideline sets out the evidence and the best recommendations for treating caries and thus aims to be an important aid in reaching the goal. The contents of the Guideline should be updated on a regular basis and at intervals of no more than five years to match new evidence and knowledge and keep up with academic and clinical advances and the changing demands of society.

\section{Materials and Methods}

\section{Outline of methods for developing the Guideline}

The Guideline was developed by a committee of nine university clinicians and a librarian. A chairperson was chosen at the biannual meeting of the Japanese Society of Conservative Dentistry (JSCD). University clinicians certified as specialists in restorative dentistry by JSCD were then selected by the chairperson and approved by the JSCD general meeting. The committee selected the most frequent clinical questions encountered in treating caries and used electronic databases to search and assess the best available scientific evidence for each question. We added lessons from clinical experience of committee members and many hours of face-to-face discussions and exchanges of e-mails to achieve a consensus in treating 
caries with MI policy. A graded recommendation was made for each clinical question. The provisional Guideline was strengthened after reviews and further discussion with university researchers and general practitioners.

\section{Committee members}

All nine university clinicians were experts in conservative dentistry, as certified by JSCD and actively engaged in teaching students and clinical work, and six also had dental materials senior advisors certificates from the Japanese Society for Dental Materials and Devices.

\section{Clinical questions addressed in developing the guideline}

The committee selected the most frequent clinical questions (CQs) encountered in treating caries. Committee members reached consensus that the Guideline for treating caries should cover a wide range of areas including remineralizing lesions, which had not produced cavities, removing infected dentin, sealing exposed dentin, and restoring defects. Since treatments of caries obviously involve large numbers of topics, the committee decided to focus on those caries which needed removal. Nevertheless, remineralizing treatment for root caries was included since it is an increasingly important concern as Japan's population ages. Restorative treatments after removing carious dentin were included as a sequential part of the treatments of caries.

\section{Systematic review methods for clinical questions}

\section{i) Selection criteria of literatures}

Inclusion and exclusion criteria for the selection of papers for the Guideline were established prior to the literature search. The inclusion criteria consisted of written clinical studies: systematic reviews, randomized controlled trials (RCTs) and controlled clinical trials (CCTs), in which the diagnoses and treatments of caries were described. In the event, there were few RCTs and CCTs found, so case series were also considered. Since this Guideline is focused on permanent teeth, primary teeth were excluded. Case reports and laboratory studies were included only where the higher literature fell short.

\section{ii) Search strategy}

The journal search, consisting of both electronic and manual searching, was 
undertaken to identify all relevant studies written in English and Japanese.

1) Electronic databases

An electronic search was conducted by the librarian member of the committee, and included the following databases from 1970 to 2008: PubMed, MEDLINE and Igaku Chuo Zasshi (Japanese). The subject search used a combination of controlled vocabulary and free text based on the search strategy for PubMed and MEDLINE (http://wwwsoc.nii.ac.jp/jscd/mem). The literature search in Japanese was also conducted using the same search strategy.

2) Manual searching

The reference lists of all the relevant studies, existing reviews and personal reprint collections of authors were screened for additional relevant publications.

\section{iii) Selection of relevant publications}

Each publication was initially assessed for relevance by two members using the information presented in the abstract. When an abstract was not available or failed to provide sufficient information, a reprint of the full paper was obtained. When papers or abstracts reported different stages of clinical trials, only the longer-term study was included in the review.

\section{iv) Evaluation of clinical effectiveness}

1) Study selection

Systematic Reviews, RCTs and CCTs, in which the clinical effectiveness of diagnosing and treating caries in permanent teeth were evaluated and discussed, were selected by two members acting independently for each clinical question. When few RCTs and CCTs were found, relevant case series were included. Basically, case studies and laboratory studies were excluded. The study design of each of the selected papers was assessed by the two reviewers independently in the review process. If a trial was excluded from evaluation, the reasons for exclusion were described.

2) Data extraction and synthesis

The following information was extracted from the papers selected for evaluation of the clinical effectiveness of diagnosing and treating caries in permanent teeth: date of the study, year of publication, setting and funding source of the trials, sample size, age and gender of the patients, types of teeth and cavities restored, methods of diagnoses and treatments. Information on adverse events or effects was also recorded. Significant outcomes for each CQ were identified by discussion among the committee. Data were extracted by two members 
independently. Data that could be presented in graphs and figures were extracted whenever possible. Such data were only included, however, if both reviewers independently extracted the same result. Any disagreement was discussed, and a third reviewer was consulted as necessary.The basic information and the outcome of the included papers were summarized in tables for each CQ with the order of the level of evidence (Table 1). Then, structural abstracts were made for papers, which were identified as the best available clinical evidence.

\section{Grading recommendations}

A graded recommendation (Table 2) was made for each clinical question. The principles of Delphi Method were applied in reaching consensus and deciding grading recommendations for each CQ after discussing the level of clinical evidence, the possible positive impact on the Japanese clinical situation, the cost of the treatment, adverse events, and the lessons from the clinical experience of committee members.

Table 1. Level and relevant research designs

Levels of evidence

I Systematic review / meta-analysis of randomized controlled trials (RCTs)

II RCTs

III Controlled non-randomized trials

IV Analytic, epidemiological studies (cohort study, case-control study, cross-sectionalstudy)

V Descriptive studies (case reports, case series)

VI Opinions of experts and expert groups based on experience rather than on clinical data

Table 2. Recommendations listed by grade*

Grade and Description

Grade A Strongly recommended on sound scientific evidence (Level II or above)

Grade B Recommended on scientific evidence (Level III)

Grade C1 Recommended in spite of a lack of high-level scientific evidence

Grade C2 Not recommended due to insufficient scientific evidence

Grade D Not recommended given ineffective or adverse effects indicated by the scientific evidence 
(*Based on consensus by the expert working group supported by bibliographic search and guidelines proposed by Minds ${ }^{2)}$.

\section{External reviewing}

The provisional Guideline was reviewed by nine external reviewers who were three university researchers, five general practitioners and one dentist employed by a company. A specialist of evidence-based medicine was included. They were asked to review the provisional Guideline based on the $\mathrm{AGREE}^{3}$ instrument and to provide any comments from their clinical experiences. The committee discussed the feedbacks from the reviewers and the Guideline was strengthened after input and discussion with the reviewers.

\section{Results}

The Guideline addresses the 16 most frequent clinical questions (CQs) encountered in treating caries, basically according to the depth of caries. In addition, questions on restorative methods and root caries were included.

\section{Examination and diagnosis of primary caries and decision on intervention}

CQ1: What examinations are effective for the diagnosis of occlusal caries? Recommendation: Where a cavity has clearly already formed, visual examination and probing are effective. In cases without cavity formation, including so-called hidden caries*, radiographic examination should be done (Level I ${ }^{4-6}$ ). (Grade of recommendation A)

* Hidden (occult) caries refers to lesions that may be overlooked even when the operative field is very carefully examined after being cleaned and dried; X-rays may reveal extensive decalcified tooth lesions.

CQ 2: What examinations are effective for the diagnosis of proximal caries? Recommendation: Where a cavity has formed, visual examination and probing are effective. In cases without cavity formation, bitewing radiographic examination or transillumination testing is effective (Level ${ }^{4-6}$ ). (Grade of recommendation A)

CQ 3: How far must caries progress before cavity preparation is indicated?

Recommendation: A restoration is indicated whenever the following findings are noted. Intervention should be done immediately where more than one of findings are evident (Level 
$\left.\mathrm{VI}^{7-11}\right)$. (Grade of recommendation B)

1) A cavity is visually detected after cleaning and drying the tooth.

2) There is pain or discomfort from cold water or food-impaction.

3) There is unacceptable appearance.

4) X-rays reveal lesions penetrating more than a third of the dentin.

5) A patient is at high risk of caries.

\section{Extent of caries removal in cavities of intermediate depth}

CQ 4: Are hardness and color reliable diagnostic criteria in determining how much carious tissue should be removed?

Recommendation: The microbial count in cases of hard carious dentin is significantly lower than in soft carious dentin (Level $\mathrm{V}^{12}$ ). On the other hand, when strongly discolored carious dentin is removed, a transparent layer free from microbial infection, and ranging from amber to flaxen, is observed (Level $\mathrm{V}^{13}$ ). Accordingly, it is recommended that carious dentin should be removed using a sharp spoon excavator ${ }^{14,15}$ or a round bur at low speed, taking both hardness and color into consideration. (Grade of recommendation C1)

CQ 5: In the removal of carious dentin, should caries detector dyes be used ? Recommendation: By using caries detector dyes, infected dentin can be removed securely, and excessive tooth preparation can be avoided (Level $\mathrm{V}^{13}$ with use of $1 \%$ acid red propylene glycol solution ${ }^{\text {a)}}$; Level VI ${ }^{16}$ with use of $1 \%$ acid red polypropylene glycol solution ${ }^{\text {b) }}$ ). Accordingly, for removal of carious dentin, use of caries detector dyes is recommended. (Grade of recommendation B)

\section{Pulp protection in deep caries}

CQ 6: Is a base required under a resin composite restoration?

Recommendation: In cases of deep cavities * with no pulp exposure restored with a resin composite through reliable bonding systems**, the presence or absence of a lining or base does not affect the incidence of postoperative pulp symptoms (Level II ${ }^{17-19}$ ). Accordingly, in the restoration of deep caries with a resin composite, a lining or base is not required. (Grade of recommendation $\mathrm{B}$ )

* Deep cavities mean one that extends into two-thirds of the dentin as shown by a radiograph. 
**Reliable bonding systems refer to recently developed self-etching bonding systems. Research ${ }^{19}$ has shown that such systems are effective in producing long-lasting restorations with the caveat that the procedures are technically sensitive. It depends on appropriate removal of carious dentin, the cavity being isolated by a rubber dam, and the bonding system being precisely applied to the cavity.

\section{Treatments of deep caries where there is high risk of pulp exposure - cases of clinically healthy pulp or reversible pulpitis}

CQ 7: Can pulp exposure be avoided by using step-wise excavation?

Recommendation: In cases where deep caries have penetrated the pulp, pulp exposure can nevertheless be avoided by step-wise excavation, if the pulp is clinically healthy or shows symptoms of reversible pulpitis (Level II ${ }^{20,}{ }^{21}$ ). Accordingly, step-wise excavation is recommended. (Grade of recommendation B)

CQ 8: In cases where step-wise excavation is performed, are pulpal symptoms the same as in the case of complete removal of caries?

Recommendation: Where deep caries have penetrated the pulp, provided the pulp is clinically healthy or shows symptoms of reversible pulpitis, step-wise excavation maintains the same pulpal conditions as in cases of complete caries removal where the pulp is not exposed (Level II ${ }^{20,21}$ ). Accordingly, step-wise excavation is recommended. (Grade of recommendation B)

CQ 9: Which pulp capping agents are appropriate for step-wise excavation?

Recommendation: In cases of deep caries that have affected the pulp, if the pulp is clinically healthy or manifests symptoms of reversible pulpitis, step-wise excavation using a calcium hydroxide or polycarboxylate cement containing tannin/fluoride compound can reduce caries-related bacteria (Level III ${ }^{22-28}$ ). It can also harden carious dentin with use of calcium hydroxide (Level $\mathrm{V}^{25-28}$ ); or with use of polycarboxylate cement containing tannin/fluoride compound (Level III ${ }^{24}$ ). Accordingly, a calcium hydroxide or polycarboxylate cement containing tannin/fluoride compound is recommended for use in step-wise excavation. (Grade of recommendation B) 
CQ 10: In step-wise excavation, approximately how much time should elapse before re-entry?

Recommendation: In cases of deep caries that have affected the pulp, if the pulp is clinically healthy or manifests symptoms of reversible pulpitis, after performing step-wise excavation using a calcium hydroxide or polycarboxylate cement containing tannin/fluoride compound, hardening of carious dentin usually occurs after three to 12 months (Level $\mathrm{V}^{25-28}$ with use of calcium hydroxide; Level III ${ }^{24}$ with use of polycarboxylate cement containing tannin/fluoride compound). Accordingly, when step-wise excavation has been performed using a calcium hydroxide or polycarboxylate cement containing tannin/fluoride compound, re-entry is recommended after three months, at which time residual infected dentin should be removed. (Grade of recommendation B)

\section{Usefulness of direct resin composite restorations for posterior teeth}

CQ 11: Are there any differences in clinical results between direct resin composite restorations and metal inlay restorations on the occlusal surfaces of posterior teeth?

Recommendation: There are no significant differences in clinical results between direct resin composite restorations and metal inlay restorations on the occlusal surfaces of posterior teeth (Level $\mathrm{V}^{29,30}$ ). However, in direct resin composite restorations, since the removal of caries is performed on the basis of MI and using reliable bonding procedures, greater sound tooth structure can be preserved. In addition esthetic restorations can be performed. Accordingly, direct resin composite restorations are recommended for occlusal surfaces. (Grade of recommendation B)

CQ 12: Are there any differences in clinical results between direct resin composite restorations and metal inlay restorations on the proximal surfaces of posterior teeth?

Recommendation: There are no significant differences in clinical results between direct resin composite restorations and metal inlay restorations on proximal surfaces of posterior teeth (Level $\mathrm{V}^{29}, 30$ ). However, in direct resin composite restorations, since the removal of caries is performed on the basis of MI, greater sound tooth structure can be preserved, and esthetic restorations can also be performed. Accordingly, provided that the conditions are met for reliable bonding and filling procedures to be performed adequately ${ }^{31}$, direct resin composite restorations are recommended for proximal surfaces of posterior teeth. (Grade of recommendation $\mathrm{C} 1$ ) 


\section{Merits of repair-repairing and refurbishing}

CQ 13: In cases of resin composite restorations where marginal discolorations or defects are observed, is repair as effective as replacement?

Recommendation: In cases of resin composite restorations where marginal discolorations or defects are observed, repairing or using sealant offers comparable effectiveness to replacement (Level III ${ }^{32,33}$ ). No consistent results have been obtained concerning the effects of refurbishing (Level III ${ }^{32,33}$ ). However, refurbishing may be worth trying since it is minimally invasive to teeth. Accordingly, repair is recommended as a procedure that encourages preservation of sound tooth structure. (Grade of recommendation B)

CQ 14: In cases of resin composite restorations where secondary caries is observed, is repair as effective as replacement?

Recommendation: In cases of secondary caries found in resin composite restorations, there is little clinical research on the effectiveness of restoration repair. However, the expert working group reached a consensus based on the extensive clinical experiences of its members (Level VI). This is that if the secondary caries can be completely removed, and repairing can be made in an appropriate environment, repair is recommended not only from the standpoint of tooth preservation, but also of reducing the stress and burden on the patient. (Grade of recommendation $\mathrm{C} 1$ )

\section{Treatment options for root surface caries}

CQ 15: In early-stage active root surface caries, are non-invasive treatments using fluorides effective?

Recommendation: By using both toothpaste containing fluoride and mouthwash containing $0.05 \% \mathrm{NaF}$ on a daily basis, the early-stage active root surface caries can be remineralized and altered into an inactive caries (Level II ${ }^{34,35}$ ). When using only 1,100 ppm or more of fluoride-containing toothpaste, if the caries erosion depth is no more than $0.5 \mathrm{~mm}$, remineralization is still possible (Level III ${ }^{36}$ ). Accordingly, in cases of early-stage active root surface caries in which the defect is shallow, it is recommended that non-invasive treatment employing fluorides should first be attempted to promote remineralization and manage the caries ${ }^{34-39}$. (Grade of recommendation B) 
CQ 16: Is resin composite or glass-ionomer cement preferable for restoration of root surface caries?

Recommendation: In terms of marginal integrity or secondary caries, no significant differences in clinical results could be noted after one year between resin composite and glass-ionomer cement restorations when applied to root surface caries (Level III ${ }^{40}$ ). Accordingly, it is recommended that resin composite restoration be used under conditions that allow the bonding system to work effectively; glass-ionomer cement is recommended when the caries has expanded to subgingival area and moisture control is difficult. (Grade of recommendation $\mathrm{C} 1$ )

\section{Discussion}

Members of the working group devising the Guideline used their combined more than 250 years of academic study and practical clinical experience of the treatment of caries to prepare a list of the most common clinical issued confronting dentists. They then used the principles of the Delphi Method, in 100 hours of face to face discussions and more than a thousand e-mail exchanges to assess the scientific evidence and reach consensus on how best to address them. Based on this, the group devised recommendations and guidance offering the optimum course of treatment for each. Throughout the discussions, the working group sought to use the latest scientific evidence, modern materials and methods, keeping in mind the primary need for patient-centered care based on the MI concept.

\section{Examination and diagnosis of primary caries and decision-making on interventions}

\section{(CQs 1-3)}

Recent advances in clinical cariology have allowed clinicians to diagnose caries at an earlier stage, and to manage them without the need for surgical intervention. The proposals of ICDAS II (International Caries Detection and Assessment System) ${ }^{41}$ focus on early and detailed diagnosis of caries, and recommend changes in the standard restorative treatment. Early diagnosis of coronal caries in permanent teeth is important, especially in patients aged 7-18 because of the frequent onset and rapid spread of lesions in maturing teeth. However, it is often difficult to recognize hidden caries on occlusal surfaces and initial proximal caries, so clinicians do not always agree about when and how to intervene surgically ${ }^{42}$. The aim was to offer guidance and recommendations to aid better detection and subsequent decision-making about restorative treatment. 
Visual inspection, explorer probing, bitewing radiographs, electrical conductivity, fiber-optic trans-illumination (FOTI), and laser fluorescence are currently used for the diagnosis of dental caries. Several studies review the validity and reliability of these methods for the detection of occlusal and/or proximal lesions with or without cavities ${ }^{-6}$. Visual inspection and explorer probing are effective for the detection of cavitated lesions with high sensitivity. The combined use of visual inspection and radiographs remains a valid approach for the detection of non-cavitated lesions. Trans-illumination using a dental chair-mounted light source is also effective for the detection of non-cavitated proximal lesions. In Japan, visual inspection, explorer probing and radiographs are most commonly used. Devices that use electrical conductivity and FOTI would be additionally helpful, but are not commercially available yet. At present, laser fluorescence devices are not common in Japan.

There is not much high quality scientific evidence to aid decision making regarding surgical interventions in CQ3 (VI ${ }^{7-11}$ ). Because a tooth once drilled cannot be undrilled, the working group decided to upgrade the recommendation from $\mathrm{C} 1$ to $\mathrm{B}$ to delay surgical intervention until the lesion is clearly visible in the dentin ${ }^{7}$. Restorative treatment should give priority to a patient's esthetic demand whenever one or more conditions are evident. Three surveys, one conducted in Brazil ${ }^{9}$ and two in Scandinavia ${ }^{10,11}$, showed that most clinicians decide to intervene surgically when the lesion reaches the middle or the outer third of the dentin as shown by radiographs.

Attempts to reach consensus on the questions of surgical interventions included considering common practice worldwide ${ }^{43,44}$ as well as the postoperative effectiveness, and patient's burden and satisfaction with caries treatment. The consensus was that lesions deeper than the outer third of dentin shown by radiographs should be promptly treated.

\section{Extent of caries removal in cavities of intermediate depth (CQs 4 and 5)}

It is important to distinguish between caries-infected dentin, which must be removed, and dentin that is only affected by caries and should be preserved ${ }^{45}$. However, hitherto there have been no clear criteria for differentiation. To help distinguish between the two, recommendations for CQ 4 and 5 were formulated.

The recommendation for CQ 4 was based on the findings of two clinical studies that have demonstrated a close relationship between hardness ${ }^{12}$ and color ${ }^{13}$ of carious lesions and their microbial infection. The microbial count in cases of hard carious dentin is significantly 
lower than that in cases of soft carious dentin ${ }^{12}$, and a transparent layer that is free from microbial infection is observed when strongly discolored carious dentin is removed ${ }^{13}$.

Some dentists prefer not to remove even strongly discolored carious dentin (A or B in Figure 1) if the carious dentin is hard. The working group did not reach a consensus on whether such carious dentin should be removed; half of the members advised the removal of such carious dentin while the other half did not. But there was agreement that based on the color chart, lesions colored C and D should not be removed, as shown in Figure 1.

Two additional studies ${ }^{14,15}$ have shown the usefulness of a spoon-excavator with a sharp cutting edge for selective removal of caries-infected dentin while preserving the caries-affected dentin. One study ${ }^{14}$ demonstrated that the hardness of caries-infected dentin was less than 20 Knoop Hardness Numbers. It is important to use an excavator with a sharp edge, because another study ${ }^{15}$ showed that when a sharp excavator was used the hardness of the remaining dentin was 24 .

The recommendation for CQ 5 is to use one of two caries detector dyes: $1 \%$ acid red in propylene glycol solution ${ }^{13}$ or $1 \%$ acid red in polypropylene glycol solution ${ }^{16}$. One clinical study ${ }^{13}$ showed that after using the propylene glycol dye, dentin that was only palely-stained was a decalcified layer with no microbial infection and should be left to prevent excessive removal of caries-affected or healthy dentin. Another study ${ }^{16}$ showed that when using the polypropylene glycol, the dye exclusively penetrated into caries-infected dentin alone. Thus, the staining indicated caries-infected dentin, which should be completely removed, leaving sclerotic dentin to be preserved.

The evidence levels of the two studies adopted for the recommendation of using the two caries detector dyes were "V" and "VI". The working group nevertheless upgraded the recommendation from " $\mathrm{C} 1$ " to " $\mathrm{B}$ ", because there are currently no objective diagnostic criteria superior to dye-staining tests to distinguish between infected dentin, that should be removed, and caries-affected dentin, that should be preserved. Figure 2 shows stained dentin after using the dye containing $1 \%$ acid red in propylene glycol solution. A consensus was reached on the removal of dye-stained dentin.

\section{Pulp protection in deep caries (CQ 6)}

Technological innovations have led to improvements that allow adhesive composite restorations to achieve strong adhesion and good marginal sealing. These advances have demonstrated that the actual cause of pulpal irritation in restorations is microleakage and 
subsequent bacterial infection ${ }^{46,47}$. Some studies ${ }^{48,49}$ have found that directly capped pulp with adhesives can help in healing with dentin-bridge formation. As the biological compatibility of modern adhesive systems is becoming widely understood, the clinical use of traditional liners and bases of adhesive restorations has been dramatically reduced. Instead, adhesives are applied directly to dentin without any means of pulpal protection or indirect pulp capping. In spite of this, many clinicians still often place a lining or base when restoring deep caries with a resin composite because of concerns about pulpal irritation. A clinical study of endodontic complications after composite restorations demonstrated that dentin and pulp protection by conditioning-and-sealing with adhesive resins is as effective as using a conventional calcium hydroxide lining ${ }^{17}$. Short- and long-term assessments of composite restorations that use self-etching adhesives found that the absence of conventional protective layers was not responsible for pulp complications, even in deep cavities ${ }^{18,19}$. Therefore, the working group consensus was that a lining or base is not required in the restoration of deep caries with modern resin composite and reliable bonding systems.

\section{Treatments of deep caries with high risk of pulp exposure (CQs 7-10)}

Research has shown that stepwise excavation can be effective in saving pulp in extremely deep caries in permanent teeth when there are no clinical symptoms showing irreversible pulpitis ${ }^{20}$. In addition, the amount of cariogenic bacteria was also reduced and softened dentin that was allowed to remain became harder as a consequence of remineralization. Calcium hydroxide ${ }^{25-28}$ and polycarboxylate cement combined with a tannin-fluoride preparation ${ }^{24}$ are suitable pulp capping agents since these materials have been found to reduce cariogenic bacteria and to promote remineralization. A lacuna in the various research articles is that they do not explain the methods and extent to which carious dentin is removed by stepwise excavation ${ }^{50}$. Some recent clinical studies have reported promising results that when carious dentin was left in deep cavities, the cavities were restored without re-entry ${ }^{51-53}$. However, the amount of dentin that can be safely left in the cavities was not discussed in these articles. The consensus of the working group on this issue is as follows: to remove completely peripheral carious dentin; to remove as much as possible of the caries adjacent to pulp; and to avoid pulp exposure.

Avoiding pulp exposure is important since studies suggest that the prognosis of direct pulp capping is difficult to predict ${ }^{54-56}$. In addition, caries-affected and caries-infected dentin has shown inferior adhesion with dentin bonding systems compared to sound dentin ${ }^{57-59}$. 
Therefore, removing peripheral carious dentin and achieving strong marginal adhesion is essential for protecting vital pulp from bacterial invasion and other stimuli. For long-lasting restorations after deep caries removal, marginally sound dentin is critically important since it can much more reliably adhere to restorative materials.

\section{Usefulness of direct resin composite restorations for posterior teeth (CQs 11 and} 12)

Resin composite restorations for posterior teeth have greatly advanced in Japan. However, metal inlay restorations remain popular because they are fully covered by public health insurance. The number of amalgam fillings has been drastically reduced since the early 1970s, not least because of public health concerns about Minamata disease, caused by mercury poisoning and leading to neurological damage. This encouraged Japanese dentistry to develop chemical-cured resin composites in place of amalgams. After the development of dentin bonding systems, the concept of conservative caries treatment was established by Fusayama ${ }^{60}$, and it spread nationwide. Several studies have indicated that there are no significant differences between the clinical results of resin composite restorations and those of metal inlay restorations in posterior teeth ${ }^{29,30}$. However, cavity preparation for metal inlays is still based on G.V. Black's principle, which leads to sacrificing intact tooth and often results in irritation of pulpal tissue. In resin composite restorations, caries removal is performed on the basis of the MI concept. Tooth structure can be preserved and esthetic restorations can also be performed. ${ }^{61}$ Therefore, if reliable bonding procedures and resin composite filling procedures can be performed, resin composite restorations are recommended for posterior teeth.

The consensus on the scientific evidence for CQ11 and CQ12 was based on descriptive studies (Level V) ${ }^{29-31}$, which must be considered as Grade $\mathrm{C} 1$ according to the grading system of Minds. However, the recommendation level for occlusal surface was upgraded (Grade B) by the working group because of the simplicity of direct restorations. However, the situation for proximal surfaces is more complicated. Figure 3 shows the relationship between the extension of cavities on proximal surfaces and the level of difficulty of direct restoration. If reliable bonding procedures and resin composite filling procedures can be performed, resin composite restorations are recommended for proximal surfaces. Direct restoration is strongly recommended for small cavities with proximal contact (Figure 3, left); however, direct restorations are more difficult for large cavities involving a proximal ridge (Figure 3, center and right). 


\section{Merits of repair - repairing and refurbishing (CQs 13 and 14)}

Systematic searches of the literature revealed only a few studies on the effectiveness of defective resin composite restoration repair ${ }^{32,33}$. These studies found that repair, sealant and replacement significantly improved marginal adaptations compared with cases that were not re-treated. Repair and replacement gave superior results in marginal staining when compared with the no-treatment results. However, secondary caries, a primary reason for re-treating, were not included. In addition, there was no consistency in dealing with Bravo-rated restorations, which are clinically acceptable and do not need to be treated. In addition, the quality of the statistical analysis used in the studies raised questions. The insufficient data for sample sizes for each treatment and the follow-up periods mean that the data may not be reliable. Despite these flaws, the working group upgraded the recommendations since repair is consistent with the principle of minimal intervention, and may help to preserve tooth structure and extend the longevity of restored teeth.

\section{Treatment options for root surface caries (CQs 15 and 16)}

Root surface caries are becoming an increasingly common clinical problem, especially as populations age. The prevalence of root surface caries among non-institutionalized people over the age of 60 years in Western countries and Japan has been reported to be more than 50 percent ${ }^{62}$. Restoration of root surface caries can be problematic in many cases because of proximity to the gingiva, which makes complete isolation and access for the placement of restorative materials too difficult. Considering these limitations for root restorations, there has been increasing interest in the management of root caries with minimal removal of the tooth structure. Daily use of mouth rinse and toothpaste that contains fluoride has been shown to be effective in promoting remineralization of carious lesions without surgical intervention ${ }^{34-39}$. This treatment is cost-effective, less stressful to the patient, and applicable even for people who need home-care. Monitoring the lesions in combination with regular caries risk assessment can ensure the continued benefit of such a non-surgical approach. One problem is that success depends upon the compliance of patients. Simple single-visit methods to stop the development of root surface caries in the long-term are advantageous. The clinical effectiveness of the application of antimicrobial varnishes ${ }^{63}$ or adhesives ${ }^{64}$ should be further investigated.

Restorative options for decayed lesions on root surfaces include fillings with resin composites or glass-ionomer cements ${ }^{65}$. Since resin composite restorations of coronal caries 
using recently developed dentin adhesives have shown clinical success ${ }^{29-31}$, this option can be considered as the first choice for root surface caries when isolation is feasible. Use of self-etching adhesives with simple manipulations, which can prevent contamination of the cavity, may also improve prognoses. While one recent clinical study found no significant differences between resin composite and glass-ionomer cement restorations in terms of marginal integrity or secondary caries after one year ${ }^{40}$, the long-term clinical results have not been determined. Controlled clinical trials should be conducted to evaluate the usefulness of these two materials for restorations of root surfaces in cases when moisture control is difficult. Similar trials should assess the benefits of fluoride release from glass-ionomer cements in high caries-risk patients.

\section{Closing remarks}

The aim of this Guideline is to aid decision-making by dentists and all professionals who deal with dental caries. It has been developed by a committee all of whose members have many years of clinical practice in treating common and complicated cases of initial and advanced caries and who have learned from studying the literature and from discussing how they would diagnose and treat caries in various clinical situations. The Guideline thus offers a foundation of recommendations and guidance for making decisions and treatment, but it cannot be a substitute for the judgment of experienced professionals in the actual clinical situation.

\section{Appendix}

This digest version of the guidelines was compiled by the expert working group of the Japanese Society of Conservative Dentistry (JSCD). A complete edition, entitled "Clinical guidelines for treating caries following a minimal intervention policy - Evidence and consensus based study" was published in Japanese in October $2009^{66}$. This full version is also available in a PDF file at the internet home page of JSCD (http://wwwsoc.nii.ac.jp/jscd/).

\section{References}

1) Tyas MJ, Anusavice KJ, Frencken JE, Mount GJ. Minimal Intervention dentistry - a review, FDI Commission Project 1-97; International Dental Journal 2000; 50: 1-12. 
2) Medical Information Network Distribution Service by the Japanese Ministry of Health, Labor and Welfare. (http://minds.jcqhc.or.jp/)

3) Appraisal of Guidelines for Research and Evaluation (AGREE) instrument in AGREE collaboration. (www.agreecollaboration.org)

4) Barder JD, Shugars DA, Bonito AJ. A systematic review of the performance of methods for identifying carious lesions. Journal of Public Health Dentistry 2002; 62: 201-213.

5) Barder JD, Shugars DA. A systematic review of the performance of a laser fluorescence device for detecting caries. Journal of American Dental Association 2004; 135: 1413-1426.

6) Anusavice KJ. Caries risk assessment. Operative Dentistry 2001; Supplement 6: 19-26.

7) Foster LV. Three year in vivo investigation to determine the progression of approximal primary carious lesions extending into dentine. British Dental Journal 1998; 185: 353-357.

8) Igarashi K, Ohyama A, Sasaki Y, Traoka K, Toyoshima Y, Nomura Y, Hanasaki Y, Yamada R. Evidence-based approach to restorative treatment decisions on early caries. Dental Outlook 2000; 95(1): 56 64 (Japanese).

9) Traebert J, Marcenes W, Kreutz JV, Oliveira R, Piazza CH, Peres MA. Brazilian dentists' restorative treatment decisions. Oral Health Preventive Dentistry 2005; 3: $53-60$.

10) Espelid I, Tveit AB, Mejare I, Sundberg H, Hallonsten AL. Restorative treatment decisions on occlusal caries in Scandinavia. Acta Odontologica Scandinavica 2001; 59: $21-7$. 
11) Mejare I, Sundberg H, Espelid I, Tveit B. Caries assessment and restorative treatment thresholds reported by Swedish dentists. Acta Odontologica Scandinavica $1999 ; 57: 149-154$.

12) Kidd EAM, Ricketts DNJ, Beighton D. Criteria for caries removal at the enamel-dentine junction: a clinical and microbiological study. British Dental Journal 1996; 180: 287-291.

13) Fukushima M. Adhesive resin penetration into carious dentin. The Journal of the Stomatology Society Japan 1981; 48: 362-85 (Japanese).

14) Sano H. Relationship between caries detector staining and structural characteristics of carious dentin. The Journal of the Stomatology Society Japan 1987; 54: 241-70 (Japanese).

15) Shimizu A, Torii Y. Studies on spoon excavators. Part 2 The relation between sharpness of cutting edge and ability of excavation. Japanese Journal of Conservative Dentistry 1985; 28: 690-694 (Japanese).

16) Oikawa M, Kusunoki M, Itoh K, Hisamitsu H. An experimental carious detector to stain the carious infected dentin. Dental Medicine Research 2008; 28: 7-12.

17) Whitworth JM, Myers PM, Smith J, Walls AWG, McCabe JF. Endodontic complications after plastic restorations in general practice. International Endodontic Journal 2005; 38: 409-416.

18) Unemori M, Matsuya Y, Akashi A, Goto Y, Akamine A. Self-etching adhesives and postoperative sensitivity. American Journal of Dentistry 2004; 17: 191-195.

19) Unemori M, Matsuya $Y$, Hyakutake H, Matsuya S, Goto $Y$, Akamine A. Long-term follow-up of composite resin restorations with self-etching adhesives. Journal of Dentistry 2007; 35: 535-540. 
20) Ricketts DNJ, Kidd EAM, Innes N, Clarkson J. Complete or ultraconservative removal of decayed tissue in unfilled teeth. Cochrane database of systematic reviews, Willy and son. (Online) 2006 Issue 3.

21) Leksell E, Ridell K, Cvek M, Mejare I. Pulp exposure after stepwise versus direct complete excavation of deep carious lesions in young posterior permanent teeth. Endodontics and Dental Traumatology 1996; 12: 192-196.

22) Leung RL, Loesche WJ, Charbeneu GT. Effect of Dycal on bacteria in deep carious lesions. Journal of American Dental Association 1980; 100: 193-197.

23) Goto J. Clinical histological study on the effect of indirect pulp capping on dental pulp. Japanese Journal of Pedodontics 1985; 23: 926-38 (Japanese).

24) Nagamine M. Studies on treatment of deep caries lesions utilizing polycarboxylate cement combined with tannin-fluoride preparation. Journal of Okayama Dental Society 1993; 12: 1-25 (Japanese).

25) Bjørndal L, Larsen T. Changes in the cultivable flora in deep carious lesions following a stepwise excavation procedure. Caries Research 2000; 34: 502-508.

26) Bjørndal L, Thylstrup A. A practice-based study on stepwise excavation of deep carious lesions in permanent teeth: a 1-year follow-up study. Community Dentistry and Oral Epidemiology 1998; 26: 122-128.

27) Bjørndal L, Larsen T, Thylstrup A. A clinical and microbiological study of deep carious lesions during stepwise excavation using long treatment intervals. Caries Research 1997; 31: 411-417.

28) Ogawa F, Machida Y. Clinical observation of indirect pulp capping on deep carious lesions. Shika Gakuho 1984; 84: 43-50 (Japanese). 
29) Aoyama T, Aida J, Takehara J, Morita M. Factors associated with the longevity of restorations in posterior teeth. Journal of Dental Health 2008; 58: 16-24 (Japanese).

30) Kubo S, Nakasa R, Hayashi Y. Survival rates of resin composite and cast restorations. Japanese Journal of Conservative Dentistry 2001; 44: 802-809 (Japanese).

31) Akimoto N, Takamizu M, Momoi Y. 10-year clinical evaluation of a self-etching adhesive system. Operative Dentistry 2007; 32: 3-10.

32) Gordan VV, Shen C, Riley J 3rd, Mjör IA. Two-year clinical evaluation of repair versus replacement of composite restorations. Journal of Esthetic Restorative Dentistry 2006; 18: 144-154.

33) Moncada G, Fernández E, Martin J, Arancibia C, Mjör IA, Gordan VV. Increasing the longevity of restorations by minimal intervention: a two-year clinical trial. Operative Dentistry 2008; 33: 258-64.

34) Wallace MC, Retief DH, Bradley EL. The 48-month increment of root caries in an urban population of older adults participating in a preventive dental program. Journal of Public Health Dentistry 1993; 53: 133-137.

35) Petersson LG, Hakestam U, Baigi A, Lynch E. Remineralization of primary root caries lesions using an amine fluoride rinse and dentifrice twice a day. American Journal of Dentistry 2007; 20: 93-96.

36) Baysan A, Lynch E, Ellwood R, Davies R, Petersson L, Borsboom P. Reversal of primary root caries using dentifrices containing 5000 and 1100 ppm fluoride. Caries Research 2001; 35: $41-46$

37) Papas A, He T, Martuscelli G, Singh M, Bartizek RD, Biesbrock A. Comparative efficacy of stabilized stannous fluoride/sodium hexametaphosphate dentifrice and sodium fluoride/triclosan/copolymer dentifrice for the prevention of periodontitis in xerostomic patients: a 2-year randomized clinical trial. Journal of Periodontology 2007; 78: 1505-1514. 
38) Nyvad B, Fejereskov O. Active root surface caries converted into inactive caries as a response to oral hygiene. Scandinavian Journal of Dental Research 1986; 94: 281-284.

39) Billings RJ, Brown LR, Kaster AG. Contemporary treatment strategies for root surface dental caries. Gerodontics 1985; 1: 20-27.

40) Fukushima M. Clinical evaluation of the effects to inhibit secondary caries by fluoride-releasing restoratives in high caries-risk patients. Report for Comprehensive Study on Prevention of Oral Diseases using Fluoride supported by Ministry of Health, Labour and Welfare of Japan 2004; 149-159 (Japanese).

41) ICDAS Coordinating Committee: Rationale and evidence for the international caries detection and assessment system (ICDAS II), Sept 2005. (http://www.icdas.org/)

42) Fejerskov O, Kidd E. Dental caries - the disease and its clinical management $2^{\text {nd }}$ edition. Blackwell Munksgaard, Oxford, 2008; 556-574.

43) Elderton RJ. Overtreatment with restorative dentistry: when to intervene? International Dental Journal 1993; 43: 17-24.

44) Rindal DB, Gordan VV, Litaker MS, Bader JD, Fellows JL, Qvist V, Wallace-Dawson MC, Anderson ML, Gilbert GH, For The DPBRN Collaborative Group, which includes practitionerinvestigators, faculty investigators, and staff investigators who contributed to this activity. Methods dentists use to diagnose primary caries lesions prior to restorative treatment: Findings from The Dental PBRN. Journal of Dentistry 2010; 38: 1027-1032.

45) Neves AA, Coutinho E, De Munck J, Van Meerbeek B. Caries-removal effectiveness and minimal-invasiveness potential of caries-excavation techniques: A micro-CT investigation. Journal of Dentistry 2011; 39: 154-162.

46) Brannström M, Nordenvall KJ. Bacterial penetration, pulpal reaction and inner surface of Concise Enamel Bond. Composite fillings in etched and unetched cavities. Journal of Dental 
Research 1978; 57: 3-10.

47) Fujitani M, Inokoshi S, Hosoda H. Effect of acid etching on the dental pulp in adhesive composite restorations. International Dental Journal 1992; 42: 3-11.

48) Akimoto N, Momoi Y, Kohno A, Suzuki S, Otsuki M, Suzuki S, Cox CF. Biocompatibility of Clearfil Liner Bond 2 and Clearfil AP-X System on non-exposed and exposed primary teeth. Quintessence International 1998; 29; 177-188.

49) Fujitani M, Shibata S, Van Meerbeek B, Yoshida Y, Shintani H. Direct adhesive pulp capping: Pulpal healing and ultra-morphology of the resin-pulp interface. American Journal of Dentistry 2002; 15: 395-402.

50) Hayashi M, Fujitani M, Yamaki C, Momoi Y. Ways of enhancing pulp preservation by stepwise excavation-A systematic review. Journal of Dentistry 2011; 39: 95-107.

51) Mertz-Fairhurst EJ, Curtis Jr. JW, Ergle JW, Rueggeberg FA, Adair SM. Ultraconservative and cariostatic sealed restorations: results at year 10. Journal of American Dental Association 1998; 129: 55-66.

52) Maltz M, Oliveira EF, Fontanella V, Carminatti G. Deep caries lesions after incomplete dentin caries removal: 40-month follow-up study. Caries Research 2007; 41: 493-496.

53) Thompson V, Craig GC, Curro FA, Green WS, Ship JS. Treatment of deep carious lesions by complete excavation or partial removal (A critical review). Journal of American Dental Association 2008; 12: 55-66.

54) Matsuo T, Nakanishi T, Shimizu H, Ebisu S. A clinical study of direct pulp capping applied to carious-exposed pulps. Journal of Endodontics 1996; 22: 551-556.

55) Barthel CR, Rosenkranz B, Leuenberg A, Roulet J-F. Pulp capping of carious exposures: Treatment outcome after 5 and 10 years: A retrospective study. Journal of Endodontics 2000; 26: $525-528$. 
56) Al-Hiyasat AS, Barrieshi-Nusair KM, Al-Omari MA. The radiographic outcomes of direct pulp-capping procedures performed by dental students: a retrospective study. Journal of the American Dental Association 2006; 137:1699-1705.

57) Yoshiyama M, Tay FR, Doi J, Nishitani Y, Yamada T, Itou K, Carvalho RM, Nakajima M, Pashley DH. Bonding of self-etch and total-etch adhesives to carious dentin. Journal of Dental Reseach 2002; 81: 556-560.

58) Yoshiyama M, Doi J, Nishitani Y, Itota T, Tay FR, Carvalho RM, Pashley DH. Bonding ability of adhesives resin to carious-affected and caries-infected dentin. Journal of Applied Oral Science 2004; 12: 171-176.

59) Wei S, Sadr A, Shimada Y, Tagami J. Effect of caries affected dentin hardness on the shear bond strength of current adhesives. Journal of Adhesive Dentistry 2008; 10: 431-440.

60) Fusayama T. A Simple Pain-Free Adhesive Restorative System by Minimal Reduction and Total Etching. Ishiyaku, Euro-America Inc, St Louis, Missouri, 1993; 1-22.

61) Lubisich EB, Hilton TJ, Ferracane JL, Pashova HI, Burton B, Northwest PRECEDENT. Association between caries location and restorative material treatment provided. Journal of Dentistry 2011; 39: 302-308.

62) Imazato S, Ikebe K, Nokubi T, Ebisu S, Walls AWG. Prevalence of root caries in a selected population of older adults in Japan. Journal of Oral Rehabilitation 2006; 33: 137-143.

63) Wicht MJ, Haak R, Lummert D, Noack MJ. Treatment of root caries lesions with chlorhexidine-containing varnishes and dentin sealants. American Journal of Dentistry 2003; 16: $25 \mathrm{~A}-30 \mathrm{~A}$.

64) Kuramoto A, Imazato S, Walls AWG, Ebisu S. Inhibition of progression of root caries by an antibacterial adhesive. Journal of Dental Research 2005; 84: 89-93. 
65) Burgess JO. Dental Materials for the restoration of root surface caries. American Journal of Dentistry 1995; 8: 342-351.

66) Clinical guidelines for treating caries following a minimal intervention policy -Evidence and consensus based report. Nagasue Shoten, Ltd. Kyoto, 2009.

\section{Note}

a) Caries Detector (Kuraray Medical, Tokyo, Japan )

b) Caries Check (Nihon Shika Yakuhin, Shimonoseki, Japan )

c) Dycal (Dentsply/Caulk, Milford, USA)

d) HY-Bond Temporary Cement Soft (Shofu, Kyoto, Japan) 


\section{Acknowledgement}

The committee would like to thank Professor Nairn Wilson, Dean of the King's College London Dental Institute for his support and always helpful and constructive guidance in commenting both on the facts and the felicity of the English in several drafts of this

report, which is a precis of a Guideline originally written in Japanese and running to more than 150 pages. 


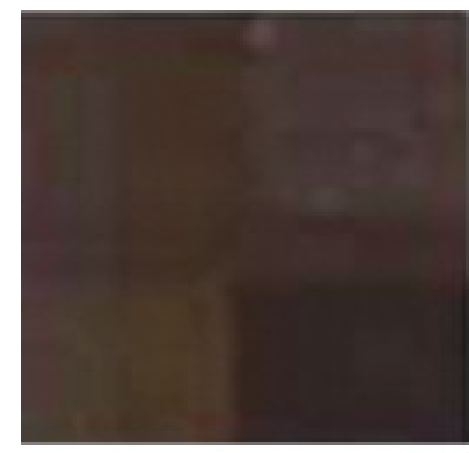

A

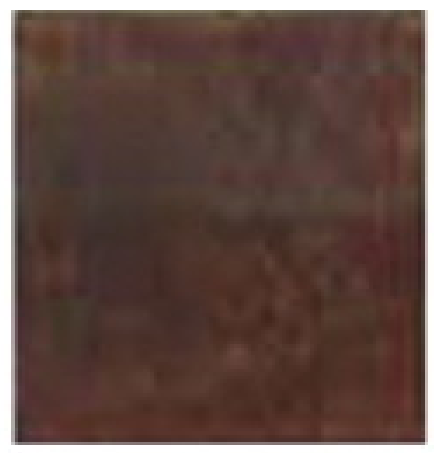

B

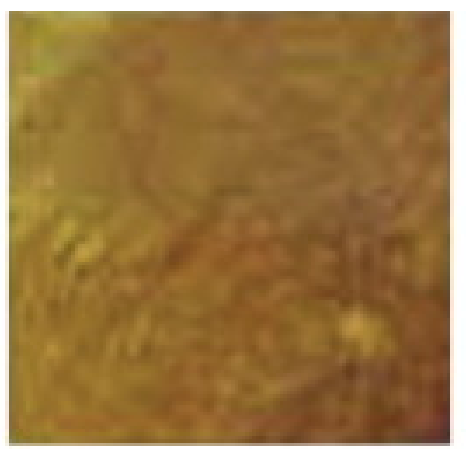

C

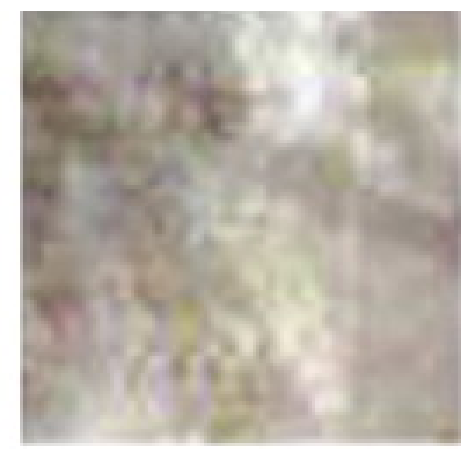

D

Figure 1. Colour samples of carious dentin 


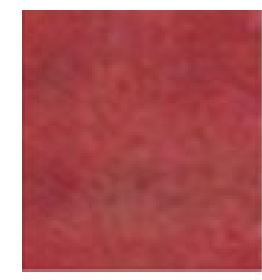



Remove


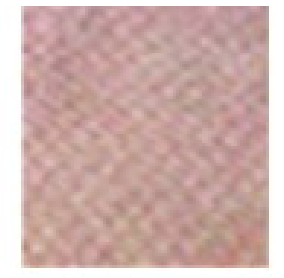

$\longleftarrow \quad$ Do not Remove

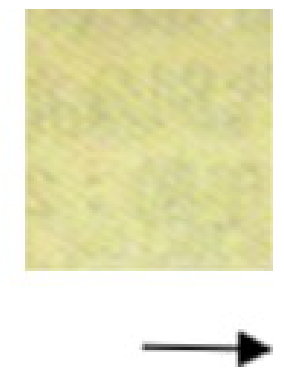

Figure 2. The expert working group agreed by consensus that removal of carious dentin was effectively indicated by the colour after staining using a dye containing $1 \%$ acid red propylene glycol. 


\section{Level of difficulty \\ Low $\longleftrightarrow$ High}

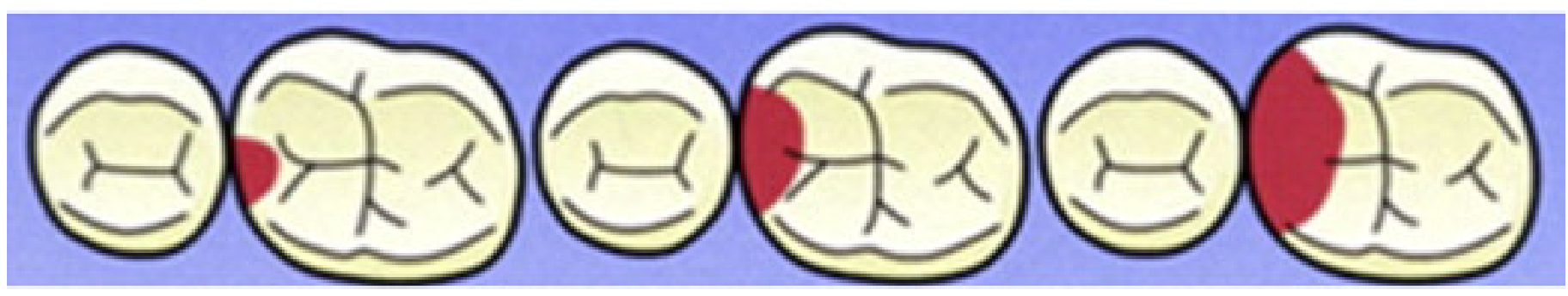

contact remained

no contact

Figure 3. Level of difficulty in resin composite restorations in relation to cavity forms.

For small cavities localized on proximal surfaces, direct restoration is simple. However, for relatively large cavities involving a proximal ridge. the direct restoration may be more difficult.(by courtesy of Dr Inokoshi) 